\title{
Efficacy of $\alpha$-blocker in improving ureteral stent- related symptoms: a meta-analysis of both direct and indirect comparison
}

This article was published in the following Dove Press journal:

Drug Design, Development and Therapy

26 May 2016

Number of times this article has been viewed

\author{
Feng $\mathrm{He}$ \\ Li-bo Man \\ Gui-zhong Li \\ Ning Liu \\ Department of Urinary Surgery, \\ Beijing Jishuitan Hospital, Beijing, \\ People's Republic of China
}

Correspondence: Feng $\mathrm{He}$

Department of Urinary Surgery, Beijing Jishuitan Hospital, Xinjiekou No 3I East Street, Xicheng District, Beijing 100035 , People's Republic of China

Tel +86 I0 585I 6688

Emailhf1998md@sina.com
Objective: To critically evaluate the efficacy of an $\alpha$-blocker in improving ureteral-stent-related symptoms and preliminarily investigate the difference between different types of $\alpha$-blockers. Methods: Relevant randomized controlled trials were identified through searching PubMed, the Cochrane Library, Embase, and other sources. After quality assessment and data abstraction, direct comparison based on the Ureteral Stent-related Symptom Questionnaire (USSQ) between $\alpha$-blockers and control was performed by RevMan 5.3. Indirect comparison between different types of $\alpha$-blockers was performed by ITC 1.0. Sensitive and subgroup analyses were used to handle important clinical factors.

Results: Sixteen randomized controlled trials containing 1,489 cases were included. Compared with control, $\alpha$-blockers significantly reduced the overall urinary symptom, pain index, general health index, and scores related to sexual matters, while no significant difference was found in work performance and additional problem scores. Subgroup analysis showed that the duration of stent insertion, patient's age, stent size, and the type of $\alpha$-blocker had the potential to influence the outcomes. Through indirect comparison, we found alfuzosin and terazosin to be better than tamsulosin in pain relief and general health improvement.

Conclusion: $\alpha$-Blocker was effective in treating ureteral stent-related symptoms, as it improved the major indexes of USSQ post-insertion or post-removal. Alfuzosin and terazosin seemed to be better than tamsulosin, which needs further verification because of the lack of direct comparison currently.

Keywords: $\alpha$-blocker, tamsulosin, alfuzosin, terazosin, ureteral stent-related discomfort

\section{Introduction}

The ureteral stent (US) was first reported in 1967 by Zimskind et al. ${ }^{1}$ Although concerted efforts to clarify its necessity were made and reduced frequency of stent placement was desired, it is still widely used in urological clinical practice to some extent as routine intervention after surgery. ${ }^{2}$ As a foreign body inserted in the ureter, part of renal pelvis, and bladder, US covers a relatively wide proportion of the urinary system and, of course, induces a series of discomforts called US-related symptoms (USSs). ${ }^{3}$ It was reported that more than half of the patients would suffer from the incidence of frequency, urgency, dysuria, and incomplete emptying, ${ }^{4,5}$ and these together with pain and other discomforts would further negatively impact the general health status, sexual activity, and work performance in $\sim 78 \%$ of cases. ${ }^{6}$ And after a US insertion, the quality of life was reported to be affected in $45 \%-80 \%$ of the patients. ${ }^{7}$

To minimize the severity of USSs, much effort has been made. Although the material, size, length, and position of the stent were fully considered and gradually 
improved, the management after stent insertion was still a longstanding challenge for both the patient and the surgeon. To prevent and treat USSs, some investigators administrated drugs including selective endogenous $\alpha$-antagonists and antimuscarinics, which are mostly used to treat urinary symptoms of benign prostatic hyperplasia. ${ }^{8,9}$ Among them, $\alpha-1$ blockers were reported to be one of the most promising drugs in patients with USSs because of their pharmacological effects of ureteral smooth relaxation and trigone inhibition. ${ }^{10}$ And, in order to investigate the efficacy of $\alpha-1$ blockers, a series of randomized controlled trials (RCTs) were conducted in the past decade. ${ }^{11-26}$ However, since they were limited to small sample sizes and patient characteristics, the therapeutic effects were varied and thus different conclusions were drawn. Therefore, in this study we comprehensively gathered all the available RCTs together to clarify the overall and individual efficacy of $\alpha-1$ blockers, and preliminarily investigated some factors important to the clinician.

\section{Methods}

\section{Data sources and search strategies}

The databases PubMed, Embase, and the Cochrane Library were comprehensively searched to identify RCTs comparing $\alpha$-blocker and placebo control in preventing and treating USSs. The literature search was performed by adopting free terms and subject headings. The free terms included "alpha-blocker”, “ $\alpha$-blocker”, “alpha blocker”, "tamsulosin”, "terazosin", “doxazosin", "alfuzosin", "naftopidil", "silodosin", "ureteral stent-related symptoms", and "USS". Other sources and strategies to widen the search results included 1) screening the references and citations of the relevant RCTs, meta-analysis, and reviews; 2) manually searching the websites of clinical register centers and professional journals on urology; and 3 ) the use of some other search engine such as Google Scholar.

\section{Inclusion criteria and study selection}

The study type should be an RCT that used a random method to divide participants into different groups. And as reported, the participants should be patients who had a US (also known as double-J ureteral stent) through laparoscopic or open procedures for various underlying diseases. According to the result of randomization, additional $\alpha$-blockers including tamsulosin, alfuzosin, terazosin, and doxazosin were administrated in the treatment group ( $\alpha$-blocker group), while placebo (or not), routine antibiotics for $\sim 5$ days, and analgesic on demand were administrated in the control group. The study mainly adopted the index of Ureteral Stent-related
Symptom Questionnaire (USSQ) as outcome measure. The USSQ is a specific USS scoring system and has six sections. Primary outcome measures were urinary symptoms, pain index (based on a visual analog scale from 1 to 10 at four locations in women and five locations in men), and general health; and secondary outcomes were work performance, sexual matters, and additional problems; other indexes were quality of life score, pain, irritative symptoms score, and obstructive symptoms score. Only relevant RCTs published in English language up to November 2015 were included. Reviews, case reports, and non-RCTs were excluded.

We screened the search results strictly following the inclusion and exclusion criteria. After assessing the titles and abstracts, final selection of an articles was made based on full-text evaluation.

\section{Data abstraction and quality assessment}

Data were entered in a predesigned table and the statistical software by two authors independently. The following items were abstracted: article information (first author, publication year, and case), participant baseline characters (age, sex, intervention, duration of stent, stent size, and outcome measured time), and outcome measures. Methodological quality was assessed by the following fields: randomization, allocation concealment, blinding (participant and outcome assessment blinding), incomplete outcome data, selective reporting of result, and any other bias. ${ }^{27}$ The results of data abstraction and quality assessment were cross-checked to eliminate the possibility of human error.

\section{Statistical analysis}

The overall effect of $\alpha$-blocker compared to control was initially analyzed by conditional meta-analysis using RevMan 5.3 (the Cochrane collaboration, Copenhagen, Denmark). According to the clinical importance, subgroup analyis of outcome measured time (post-insertion 1-2 weeks, 3-4 weeks, and post-removal) was first conducted. Data were calculated as mean difference (MD), standard MD (SMD), or risk ratio (RR), together with their $95 \%$ confidence interval (95\% CI). Only random-effects model was adopted, as it would provide a more conservative estimate. A difference with $P<0.05$ was considered statistically significant. In order to investigate the influence of other clinical factors, we carried out sensitivity analysis according to the variables as follows: study quality (excluding poor-quality trials), age (separating patients $>50$ years and $\leq 50$ years), size of stent (separating diameter $<6 \mathrm{~F}$ and $\geq 6 \mathrm{~F}$ ), and the type of $\alpha$-blockers (separating tamsulosin and other $\alpha$-blockers). 
To preliminarily investigate the difference of $\alpha$-blockers where direct comparison study was currently lacking, we made an indirect comparison of network analysis between tamsulosin and other $\alpha$-blockers using ITC 1.0 (Canadian Agency for Drugs and Technologies in Health, Ontario, Canada) due to the limited number of available RCTs.

\section{Results}

\section{Study characteristics}

The primary search yielded 296 citations. After screening the titles and abstracts, full texts of 24 citations were evaluated, and finally a total of 16 RCTs were included (Figure 1). The meta-analysis contained 1,489 cases, of whom 772 patients were in the $\alpha$-blocker group and 717 patients were in the control group. The baseline characteristics are presented in Table 1. The included studies covered four types of $\alpha-1$ blockers: tamsulosin in ten trials, ${ }^{12,14-17,21,23-26}$ alfuzosin in four trials, ${ }^{11,13,20,24}$ terazosin in two trials, ${ }^{18,22}$ and doksazosin in one trial. ${ }^{19}$ The above drugs used in most trials were within the recommended dose of $0.4 \mathrm{mg} / \mathrm{d}, 10 \mathrm{mg} / \mathrm{d}, 2 \mathrm{mg} / \mathrm{d}$, and $4 \mathrm{mg} / \mathrm{d}$, and the duration of drug administration was in accordance with the duration of the stent, which ranged from 1 week to 6 weeks. The stent size was fixed in each trial, which ranged from $4.7 \mathrm{~F}$ to $7 \mathrm{~F}$ across trials. The length was reported in the trials to be fixed, ${ }^{15,17,22}$ or adjusted by the height and weight of the patients. The outcome measured time ranged from 3 days to 1 week, 2 weeks, and 4 weeks post-insertion of the stent, and 2-4 weeks post-removal of stent.

Methodological quality assessment showed that the overall quality was moderate (Figure 2), as four trials might have potential bias and they did not report the details of randomization and blinding. ${ }^{2,16,17,19}$

\section{Urinary symptom score}

Compared with control, $\alpha$-blockers significantly decreased the mean urinary symptom score $\left(I^{2}=94 \%, \mathrm{MD}=-3.47,95 \%\right.$ CI, $-4.58,-2.36, P<0.00001)$. Subgroup analysis according to outcome measured time showed that $\alpha$-blockers

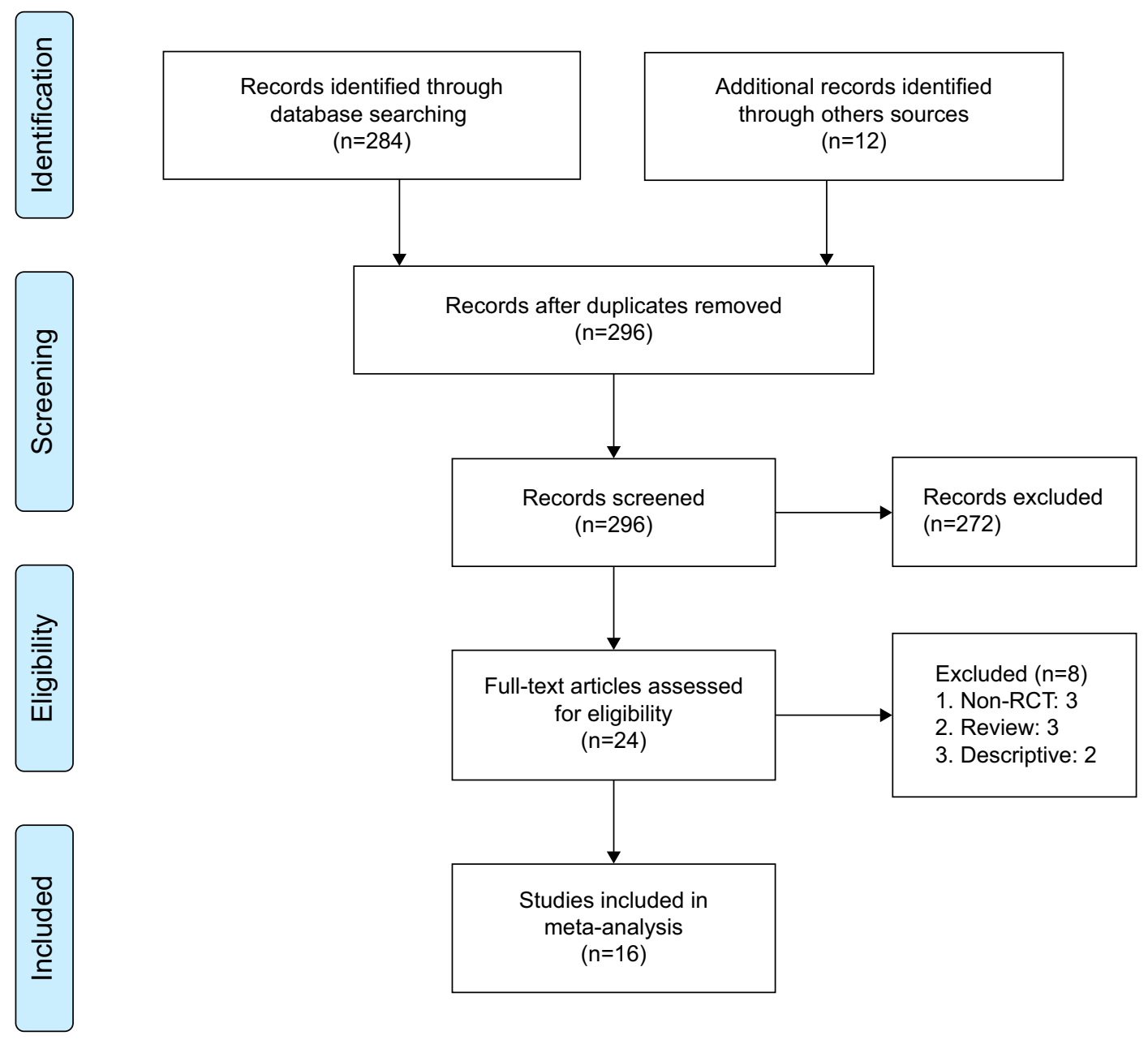

Figure I PRISMA flow diagram.

Abbreviations: RCT, randomized controlled trial; PRISMA, Preferred Reporting Items for Systematic Reviews and Meta-Analyses. 
Table I Baseline characteristics of included RCTs

\begin{tabular}{|c|c|c|c|c|c|c|c|c|c|}
\hline \multirow[t]{2}{*}{ Study } & \multirow[t]{2}{*}{ Year } & \multirow[t]{2}{*}{$\begin{array}{l}\text { Case } \\
(T / C, n)\end{array}$} & \multirow[t]{2}{*}{$\begin{array}{l}\text { Age } \\
(\mathrm{T} / \mathrm{C}, \mathrm{y})\end{array}$} & \multicolumn{2}{|c|}{$\begin{array}{l}\text { Sex (malel } \\
\text { female) }\end{array}$} & \multirow[t]{2}{*}{ Intervention (T/C) } & \multirow[t]{2}{*}{ Duration } & \multirow[t]{2}{*}{$\begin{array}{l}\text { Stent (size/ } \\
\text { length, cm) }\end{array}$} & \multirow[t]{2}{*}{$\begin{array}{l}\text { Outcome } \\
\text { measured time }\end{array}$} \\
\hline & & & & $\overline{\mathbf{T}}$ & C & & & & \\
\hline Deliveliotis et al" & 2006 & $50 / 50$ & $53.1 / 55.3$ & $30 / 20$ & $27 / 23$ & $\begin{array}{l}10 \mathrm{mg} \text { alfuzosin for } \\
4 \text { weeks/placebo }\end{array}$ & 4 weeks & $5 F / 26-28$ & PI 4 weeks \\
\hline Damiano et al ${ }^{12}$ & 2008 & $38 / 37$ & - & $29 / 46$ & & $\begin{array}{l}0.4 \mathrm{mg} \text { tamsulosin for } \\
4 \text { weeks/control }\end{array}$ & 2 weeks & $7 F /-$ & $\mathrm{PI}$ and $\mathrm{PR} I$ week \\
\hline Beddingfield et $\mathrm{al}^{13}$ & 2009 & $26 / 29$ & $45.8 / 44.0$ & $7 / 19$ & $16 / 13$ & $\begin{array}{l}10 \mathrm{mg} \text { alfuzosin for } \\
8 \text { days/placebo }\end{array}$ & $8.3 / 11.5$ days & - & PI 3 days \\
\hline Wang et $\mathrm{al}^{14}$ & 2009 & $79 / 75$ & $50.1 / 51.5$ & $63 / 16$ & $58 / 17$ & $\begin{array}{l}0.4 \mathrm{mg} \text { tamsulosin for } \\
2 \text { weeks/placebo }\end{array}$ & 2 weeks & $7 F /-$ & $\begin{array}{l}\text { PI I week, and } \\
\text { PR } 2 \text { weeks }\end{array}$ \\
\hline Wang et $\mathrm{al}^{15}$ & 2009 & $75 / 7 \mid$ & $50.4 / 50.8$ & $6 I / 14$ & $55 / 16$ & $\begin{array}{l}0.4 \mathrm{mg} \text { tamsulosin for } \\
2 \text { weeks/placebo }\end{array}$ & 2 weeks & $7 F / 26$ & PI 2 weeks \\
\hline $\begin{array}{l}\text { Navanimitkul and } \\
\text { Lojanapiwat }^{16}\end{array}$ & 2010 & $21 / 21$ & $46.1 / 51.5$ & $9 / 12$ & $6 / 15$ & $\begin{array}{l}0.4 \mathrm{mg} \text { tamsulosin for } \\
4 \text { weeks/control }\end{array}$ & 4 weeks & $6 \mathrm{~F} /-$ & $\begin{array}{l}\text { PI } 2 \text { weeks and } \\
4 \text { weeks }\end{array}$ \\
\hline $\begin{array}{l}\text { Shelbaia and } \\
\text { Elnashar }{ }^{17}\end{array}$ & 2011 & $69 / 67$ & $35.0 / 29.0$ & $44 / 25$ & $50 / 17$ & $\begin{array}{l}0.4 \mathrm{mg} \text { tamsulosin for } \\
4 \text { weeks/control }\end{array}$ & 4 weeks & $6 F / 26$ & - \\
\hline Mokhtari et al ${ }^{18}$ & 2011 & $33 / 33$ & - & - & - & $\begin{array}{l}2 \mathrm{mg} \text { terazosin for } \\
4 \text { weeks/control }\end{array}$ & 4 weeks & $4.8 \mathrm{~F} /-$ & PI 4 weeks \\
\hline $\begin{array}{l}\text { Kuyumcuoglu } \\
\text { et al }{ }^{19}\end{array}$ & 2012 & $2 \mid / 2 I$ & $45.2 / 42.9$ & $15 / 6$ & $7 / 14$ & $\begin{array}{l}4 \mathrm{mg} \text { doksazosin for } \\
4 \text { weeks/control }\end{array}$ & - & $4.7 F / 26-28$ & PI 4 weeks \\
\hline Nazim and Ather ${ }^{20}$ & 2012 & $65 / 65$ & $37.8 / 40.1$ & $52 / 13$ & $49 / 16$ & $\begin{array}{l}10 \mathrm{mg} \text { alfuzosin for } \\
\text { I week/placebo }\end{array}$ & $>$ I week & $4.7-6 \mathrm{~F} /-$ & PI I week \\
\hline Shalaby et $\mathrm{al}^{21}$ & 2013 & $82 / 81$ & $41.3 / 44$ & $55 / 27$ & $50 / 31$ & $\begin{array}{l}0.4 \mathrm{mg} \text { tamsulosin for } \\
2 \text { weeks/control }\end{array}$ & - & - & PI 2 weeks \\
\hline Tehranchi et $\mathrm{a}^{22}$ & 2013 & $23 / 24$ & $38.4 / 33.4$ & $16 / 7$ & $21 / 3$ & $\begin{array}{l}2 \mathrm{mg} \text { terazosin for } \\
13.5 \text { days/placebo }\end{array}$ & I3.5/15.4 days & $4.8 \mathrm{~F} / 28$ & PI 2 weeks \\
\hline Singh et $\mathrm{a}^{23}$ & 2014 & $30 / 30$ & $32.7 / 31.4$ & $14 / 16$ & $20 / 10$ & $\begin{array}{l}0.4 \mathrm{mg} \text { tamsulosin for } \\
4 \text { weeks/placebo }\end{array}$ & 6 weeks & $4-5 F / 24-26$ & PI 4 weeks \\
\hline Dellis et $\mathrm{a}^{24}$ & 2014 & $50 / 50$ & $45.6 / 46.9$ & $25 / 25$ & $26 / 24$ & $\begin{array}{l}0.4 \mathrm{mg} \text { tamsulosin for } \\
4 \text { weeks/placebo }\end{array}$ & 4 weeks & $6 F / 24-26$ & $\begin{array}{l}\text { PI I and } 4 \text { weeks, } \\
\text { and PR } 4 \text { weeks }\end{array}$ \\
\hline Dellis et $\mathrm{al}^{24}$ & 2014 & $50 / 50$ & $47.3 / 46.9$ & $23 / 27$ & $26 / 24$ & $\begin{array}{l}10 \mathrm{mg} \text { alfuzosin for } \\
4 \text { weeks/placebo }\end{array}$ & & & \\
\hline Park et $\mathrm{al}^{25}$ & 2015 & $20 / 23$ & $54.5 / 48.7$ & $9 / 11$ & $14 / 9$ & $\begin{array}{l}0.2 \mathrm{mg} \text { tamsulosin for } \\
2 \text { weeks/control }\end{array}$ & 2 weeks & $6 F / 20-28$ & $\begin{array}{l}\text { PI } 2 \text { weeks, and } \\
\text { PR } 4 \text { weeks }\end{array}$ \\
\hline El-Nahas et $\mathrm{al}^{26}$ & 2015 & $40 / 40$ & $41.4 / 40.8$ & $19 / 21$ & $24 / 16$ & $\begin{array}{l}0.4 \mathrm{mg} \text { tamsulosin for } \\
5 \text { weeks/placebo }\end{array}$ & 5.I/4.8 weeks & $6 F / 24-26$ & PI I-2 weeks \\
\hline
\end{tabular}

Notes: Prulifloxacin $600 \mathrm{mg}$ once daily for 5 days; ${ }^{12}$ ciprofloxacin $500 \mathrm{mg}$ twice daily for 5 days; ${ }^{17}$ patients in $\alpha$-blocker groups were administered an additional week of antibiotic therapy; ${ }^{19}$ ciprofloxacin $500 \mathrm{mg}$ twice daily for 5 days; ${ }^{23}$ and I week of oral antibiotics (one tablet quinolone two times). ${ }^{25}$

Abbreviations: RCT, randomized controlled trial; T, treatment group; C, control group; y, years; PI, post-insertion of stent; PR, post-removal of stent.

significantly improved the urinary symptom score during post-insertion $1-2$ weeks by a mean of $-3.54(95 \% \mathrm{CI},-6.54$ to -0.55$),{ }^{13,14,24-26}$ during $3-4$ weeks ${ }^{11,23,24}$ by a mean of -4.40 $(95 \% \mathrm{CI},-5.16$ to -3.64$)$, and post-removal by a mean of -2.0 (95\% CI, -2.55 to -1.46$),{ }^{14,24,25}$ as shown in Figure 3.

\section{Pain index score}

Compared with control, $\alpha$-blockers significantly decreased the mean pain index score $\left(I^{2}=98 \%, \mathrm{MD}=-0.89,95 \%\right.$ CI, $-1.15,-0.63, P<0.00001)$. Subgroup analysis according to outcome measured time showed that $\alpha$-blockers significantly reduced pain during post-insertion 1-2 weeks by a mean of $-1.58(95 \% \mathrm{CI},-3.04$ to -0.12$),{ }^{13,14,16,21,22,25,26}$ during 3-4 weeks by a mean of -0.79 (95\% CI, -1.24 to -0.34$),{ }^{11,16-18,23}$ and post-removal by a mean of -0.03 (95\% CI, -1.16 to 1.11$),{ }^{14,25}$ as shown in Figure 4.

\section{General health index score}

Compared with control, $\alpha$-blockers significantly decreased the mean general health index score $\left(I^{2}=96 \%, \mathrm{MD}=-0.50\right.$, $95 \% \mathrm{CI},-0.66,-0.34, P<0.00001)$. Subgroup analysis according to outcome measured time showed that $\alpha$-blockers significantly improved general health index score during post-insertion $1-2$ weeks by a mean of $-0.62(95 \% \mathrm{CI},-1.45$ to 0.21$),{ }^{13,14,16,24-26}$ during $3-4$ weeks by a mean of -0.62 ( $95 \% \mathrm{CI},-0.92$ to -0.33$),{ }^{11,16,17,23,24}$ and post-removal by a 


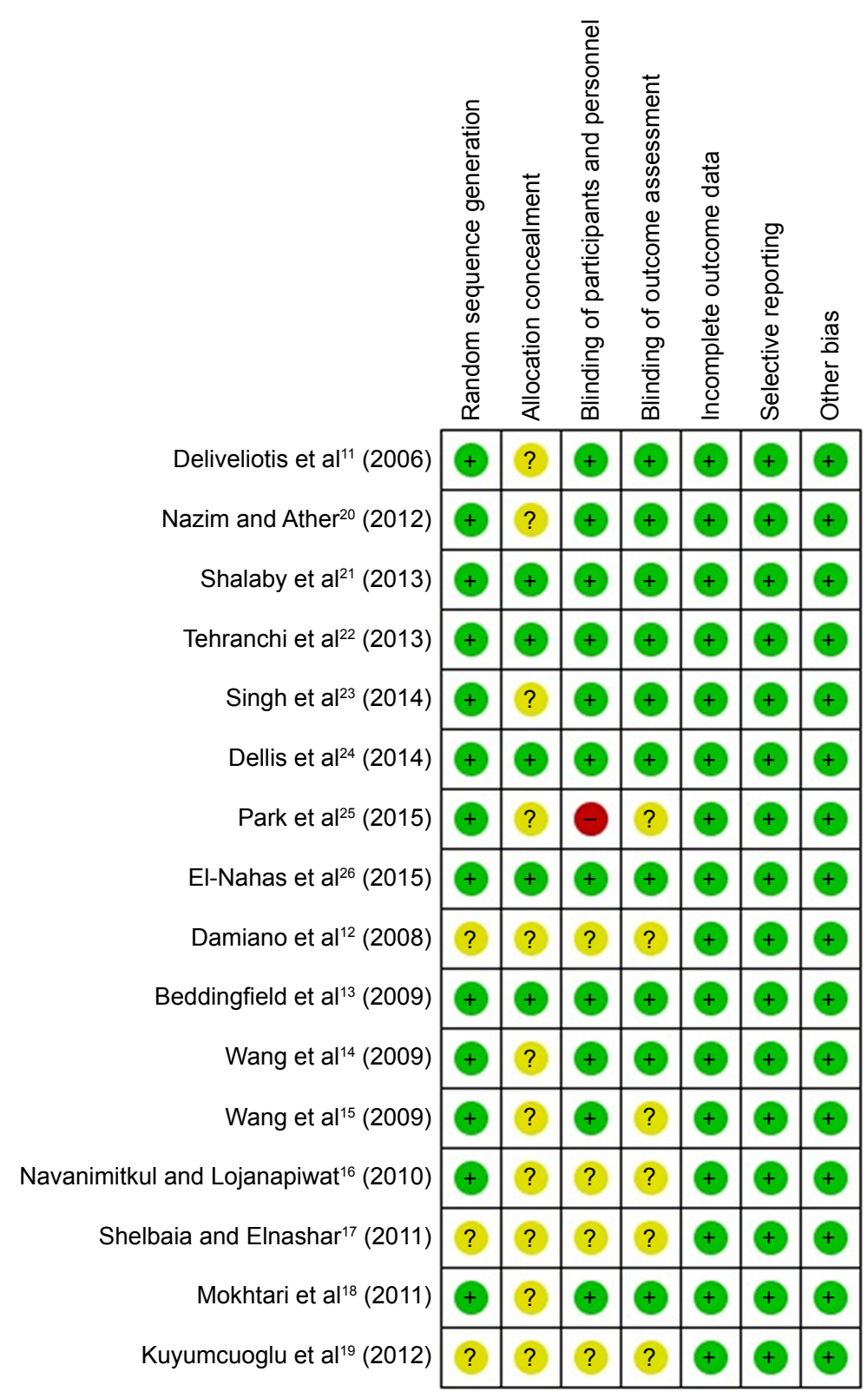

Figure 2 Methodological assessment.

mean of $-0.50(95 \% \mathrm{CI},-0.77$ to -0.23$),{ }^{14,24,25}$ as shown in Figure 5.

\section{Sensitivity analysis and network meta- analysis}

Sensitivity analysis showed that, after omitting trials with poor quality, the heterogeneity decreased. The results seemed to show that $\alpha$-blockers had a weak influence on the urinary symptom score and general health index for patients $>50$ years and on pain index for stent $\geq 6 \mathrm{~F}$. By separating tamsulosin and other $\alpha$-blockers, we found other $\alpha$-blockers seemed to have more comprehensive effects.
And, indirect comparison between tamsulosin and other $\alpha$-blockers through network meta-analysis indicated that the other $\alpha$-blockers might have a superiority in pain index score and general health score (Table 2).

\section{Work performance score}

Compared with control, $\alpha$-blockers did not induce any significant difference $\left(I^{2}=93 \%, \mathrm{MD}=1.07,95 \% \mathrm{CI},-0.15,2.30\right.$, $P=0.09)$. Subgroup analysis according to outcome measured time showed that there was no significant difference between them during post-insertion 1-2 weeks, ${ }^{13,14,25,26} 3-4$ weeks, ${ }^{23}$ and post-removal. ${ }^{14,25}$ 


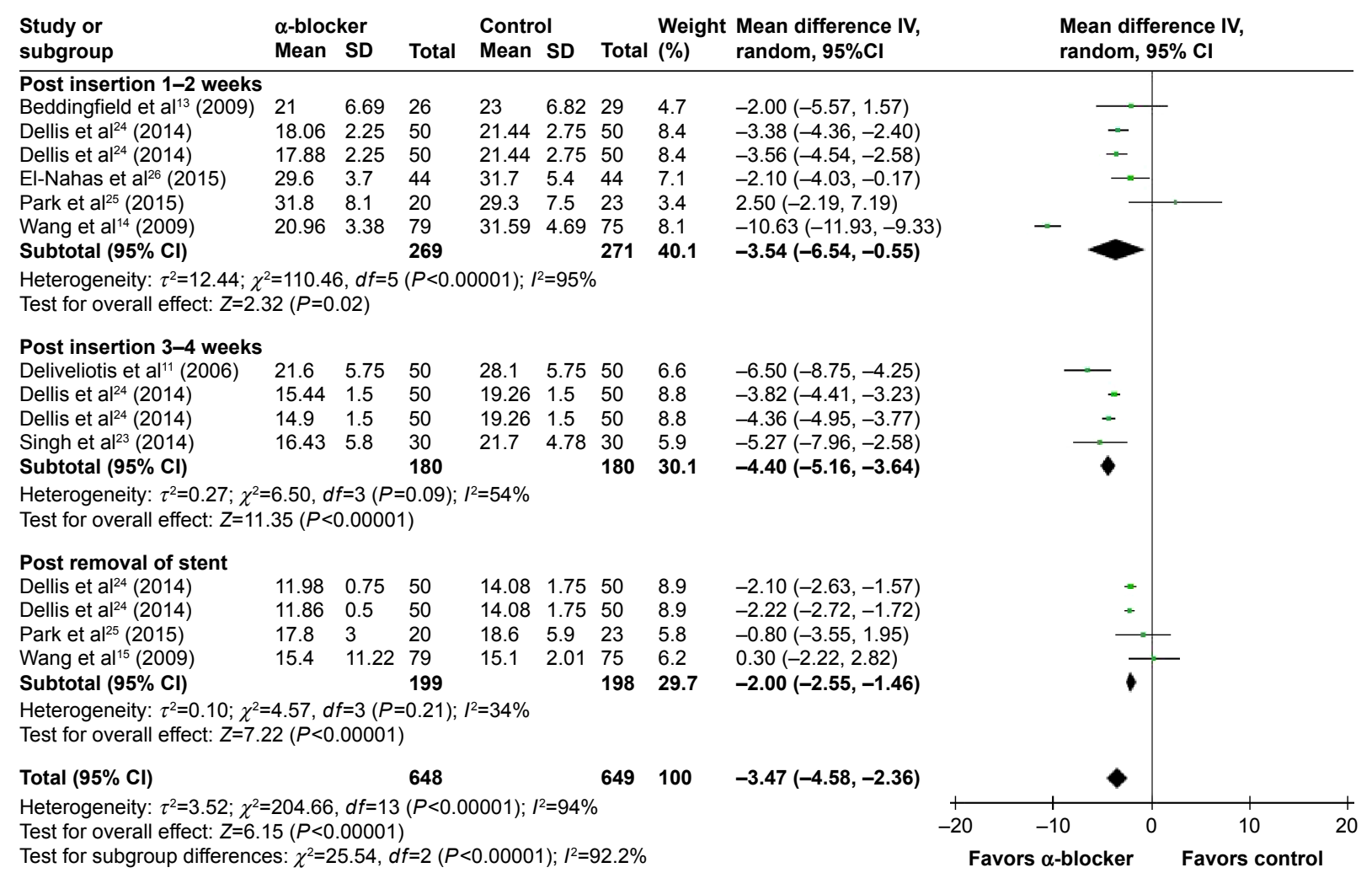

Figure 3 Meta-analysis results of mean urinary symptom score.

Abbreviations: $95 \% \mathrm{Cl}, 95 \%$ confidence interval; $d f$, degrees of freedom.

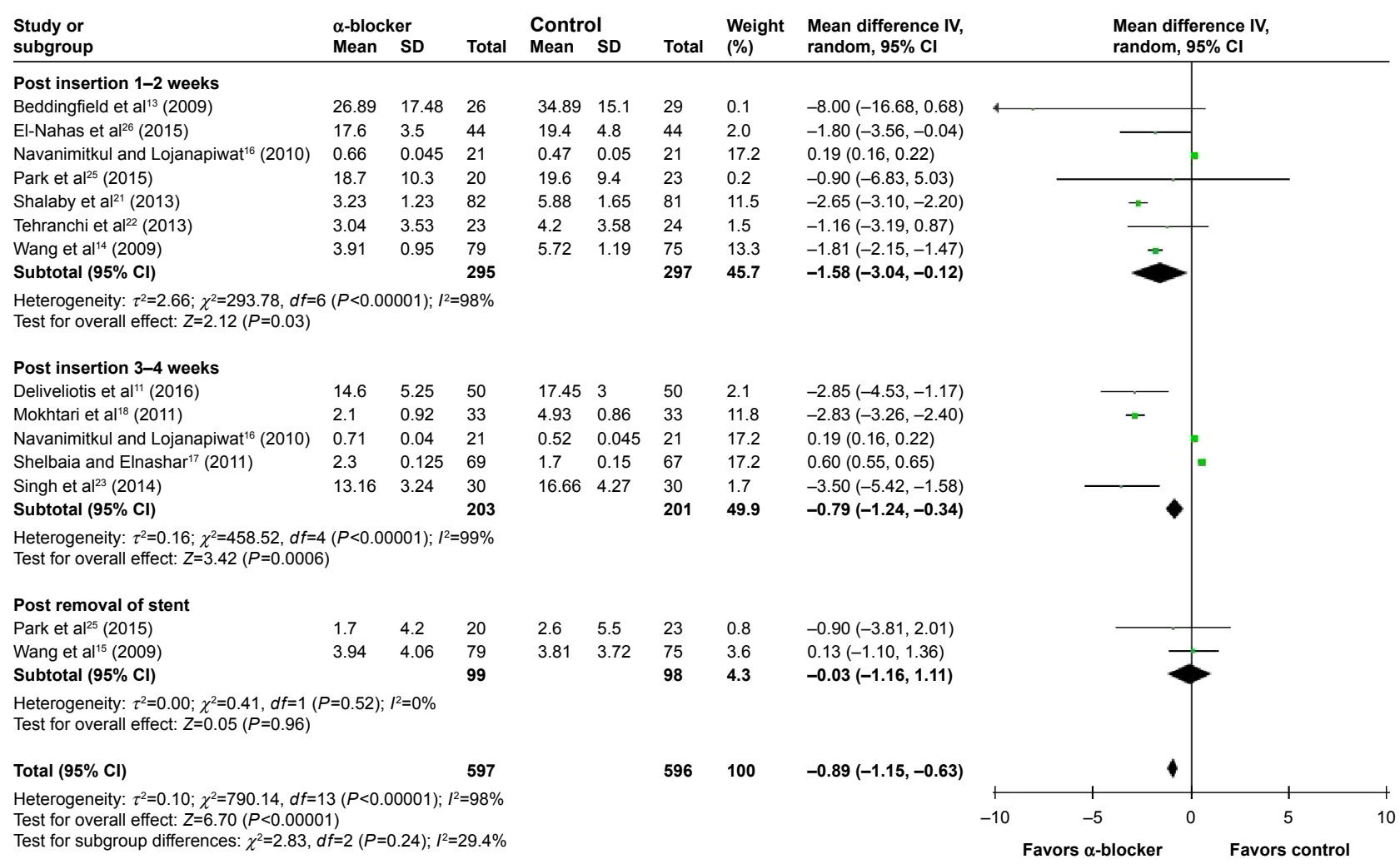

Figure 4 Meta-analysis results of mean pain index score.

Abbreviations: $95 \% \mathrm{Cl}, 95 \%$ confidence interval; $d f$, degrees of freedom. 


\begin{tabular}{|c|c|c|c|c|c|c|c|c|c|c|c|c|}
\hline \multirow{2}{*}{$\begin{array}{l}\text { Study or } \\
\text { subgroup } \\
\text { Post insertion 1-2 weeks }\end{array}$} & \multirow[t]{2}{*}{$\begin{array}{l}\alpha \text {-blocker } \\
\text { Mean }\end{array}$} & \multirow[t]{2}{*}{ SD } & \multirow[t]{2}{*}{ Total } & \multirow[t]{2}{*}{$\begin{array}{l}\text { Control } \\
\text { Mean }\end{array}$} & \multirow[t]{2}{*}{ SD } & \multirow[t]{2}{*}{ Total } & \multirow[t]{2}{*}{$\begin{array}{l}\text { Weight } \\
(\%)\end{array}$} & \multirow[t]{2}{*}{$\begin{array}{l}\text { Mean difference IV, } \\
\text { random, } 95 \% \mathrm{CI}\end{array}$} & \multicolumn{3}{|c|}{$\begin{array}{l}\text { Mean difference IV, } \\
\text { random, } 95 \% \mathrm{CI}\end{array}$} & \\
\hline & & & & & & & & & & & & \\
\hline Beddingfield et al ${ }^{13}$ (2009) & 17.27 & 4.63 & 26 & 17.41 & 4.16 & 29 & 0.5 & $-0.14(-2.48,2.20)$ & & & & \\
\hline Dellis et al ${ }^{24}(2014)$ & 8 & 1.25 & 50 & 8.92 & 2 & 50 & 4.1 & $-0.92(-1.57,-0.27)$ & & $\longrightarrow$ & & \\
\hline Dellis et $\mathrm{al}^{24}(2014)$ & 7.84 & 1 & 50 & 8.92 & 2 & 50 & 4.4 & $-1.08(-1.70,-0.46)$ & & $\longrightarrow$ & & \\
\hline El-Nahas et $\mathrm{al}^{26}(2015)$ & 16.6 & 3 & 44 & 17 & 3.4 & 44 & 1.3 & $-0.40(-1.74,0.94)$ & & & - & \\
\hline Navanimitkul and Lojanapiwat ${ }^{16}(2010)$ & 0.62 & 0.045 & 21 & 0.49 & 0.045 & 21 & 12.9 & $0.13(0.10,0.16)$ & & & 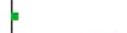 & \\
\hline Park et $\mathrm{al}^{25}(2015)$ & 15 & 6.3 & 20 & 12.2 & 4.4 & 23 & 0.2 & $2.80(-0.49,6.09)$ & & & & \\
\hline Wang et $\mathrm{al}^{14}(2009)$ & 10.1 & 2.31 & 79 & 12.2 & 2.99 & 75 & 2.8 & $-2.10(-2.95,-1.25)$ & & - & & \\
\hline Subtotal $(95 \% \mathrm{Cl})$ & & & 279 & & & 292 & 26.2 & $-0.62(-1.45,0.21)$ & & & & \\
\hline \multicolumn{13}{|c|}{$\begin{array}{l}\text { Heterogeneity: } \tau^{2}=0.88 ; \chi^{2}=54.14, d f=6(P<0.00001) ; I^{2}=89 \% \\
\text { Test for overall effect: } Z=1.47(P=0.14)\end{array}$} \\
\hline \multicolumn{13}{|l|}{ Post insertion 3-4 weeks } \\
\hline Deliveliotis et al11 (2006) & 8 & 2 & 50 & 11.4 & 2.5 & 50 & 2.6 & $-3.40(-4.29,-2.51)$ & - & & & \\
\hline Dellis et $\mathrm{al}^{24}(2014)$ & 7.04 & 0.5 & 50 & 7.98 & 1 & 50 & 8.7 & $-0.94(-1.25,-0.63)$ & & - & & \\
\hline Dellis et $\mathrm{al}^{24}(2014)$ & 6.75 & 0.5 & 50 & 7.98 & 1 & 50 & 8.7 & $-1.23(-1.54,-0.92)$ & & - & & \\
\hline Navanimitkul and Lojanapiwat ${ }^{16}(2010)$ & 0.63 & 0.048 & 21 & 0.52 & 0.045 & 21 & 12.9 & $0.11(0.08,0.14)$ & & & 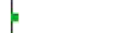 & \\
\hline Shelbaia and Elnashar ${ }^{17}$ (2011) & 2.01 & 0.15 & 69 & 1.7 & 0.15 & 67 & 12.8 & $0.31(0.26,0.36)$ & & & $=$ & \\
\hline Singh et $\mathrm{al}^{23}(2014)$ & 12.46 & 2.67 & 30 & 13.5 & 2.82 & 30 & 1.2 & $-1.04(-2.43,0.35)$ & & 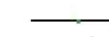 & + & \\
\hline Subtotal $(95 \% \mathrm{Cl})$ & & & 270 & & & 268 & 46.9 & $-0.62(-0.92,-0.33)$ & & $\varphi$ & & \\
\hline \multicolumn{13}{|c|}{$\begin{array}{l}\text { Heterogeneity: } \tau^{2}=0.09 ; \chi^{2}=233.65, d f=5(P<0.00001) ; I^{2}=98 \% \\
\text { Test for overall effect: } Z=4.11(P<0.0001)\end{array}$} \\
\hline \multicolumn{13}{|l|}{ Post removal of stent } \\
\hline Dellis et $\mathrm{al}^{24}(2014)$ & 6.12 & 0.25 & 50 & 6.64 & 0.5 & 50 & 11.5 & $-0.52(-0.67,-0.37)$ & & $=$ & & \\
\hline Dellis et $\mathrm{al}^{24}(2014)$ & 6.28 & 0.25 & 50 & 6.64 & 0.5 & 50 & 11.5 & $-0.36(-0.51,-0.21)$ & & . & & \\
\hline Park et al ${ }^{25}(2015)$ & 8.9 & 3.5 & 20 & 7.9 & 3 & 23 & 0.6 & $1.00(-0.96,2.96)$ & & 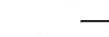 & & \\
\hline Wang et $\mathrm{al}^{15}(2009)$ & 8.24 & 1.47 & 79 & 9.59 & 3.12 & 75 & 3.2 & $-1.35(-2.13,-0.57)$ & & - & & \\
\hline Subtotal $(95 \% \mathrm{Cl})$ & & & 199 & & & 198 & 26.9 & $-0.50(-0.77,-0.23)$ & & $\boldsymbol{\varphi}$ & & \\
\hline \multicolumn{13}{|c|}{$\begin{array}{l}\text { Heterogeneity: } \tau^{2}=0.04 ; \chi^{2}=9.33, d f=3(P=0.03) ; I^{2}=68 \% \\
\text { Test for overall effect: } Z=3.64(P=0.0003)\end{array}$} \\
\hline \multirow{3}{*}{\multicolumn{3}{|c|}{$\begin{array}{l}\text { Total }(95 \% \mathrm{Cl}) \\
\text { Heterogeneity: } \tau^{2}=0.05 ; \chi^{2}=406.93, d f=16(P<0.00001) ; I^{2}=96 \% \\
\text { Test for overall effect: } Z=6.14(P<0.00001) \\
\text { Test for subgroup differences: } \chi^{2}=0.37, d f=2(P=0.83) ; I^{2}=0 \%\end{array}$}} & 759 & & & 758 & 100 & $-0.50(-0.66,-0.34)$ & & 1 & & \\
\hline & & & & & & & & & +4 & -2 & 2 & 4 \\
\hline & & & & & & & & & & $\begin{array}{l}\text { Favors } \\
\alpha \text {-blocker }\end{array}$ & $\begin{array}{l}\text { Favors } \\
\text { control }\end{array}$ & \\
\hline
\end{tabular}

Figure 5 Meta-analysis results of mean general health index score.

Abbreviations: $95 \% \mathrm{Cl}, 95 \%$ confidence interval; $d f$, degrees of freedom.

\section{Sexual matters score}

Compared with control, $\alpha$-blockers significantly decreased the mean sexual matters score $\left(I^{2}=71 \%, \mathrm{MD}=-0.29,95 \%\right.$ CI, $-0.44,-0.15, P<0.0001)$. Subgroup analysis according to outcome measured time showed that $\alpha$-blockers significantly improved sexual matters during post-insertion 1-2 weeks by a mean of $-0.42(95 \% \mathrm{CI},-0.91$ to 0.06$),{ }^{13,14,24-26} 3-4$ weeks by a mean of $-0.44(95 \% \mathrm{CI},-0.64$ to -0.24$),{ }^{11,24}$ and postremoval by a mean of -0.21 ( $95 \% \mathrm{CI},-0.35$ to -0.06$),{ }^{14,24,25}$ as shown in Figure 6.

Table 2 Sensitivity analysis and indirect-comparison meta-analysis results

\begin{tabular}{|c|c|c|c|c|c|c|c|c|}
\hline & \multicolumn{2}{|c|}{$\begin{array}{l}\text { Urinary symptom } \\
\text { score }\end{array}$} & \multicolumn{2}{|c|}{ Pain index score } & \multicolumn{2}{|c|}{$\begin{array}{l}\text { General health index } \\
\text { score }\end{array}$} & \multicolumn{2}{|c|}{$\begin{array}{l}\text { Sexual matters } \\
\text { score }\end{array}$} \\
\hline & $I^{2}$ & MD (95\% Cl) & $I^{2}$ & MD (95\% Cl) & $I^{2}$ & MD (95\% Cl) & $I^{2}$ & MD (95\% Cl) \\
\hline \multicolumn{9}{|l|}{ Sensitivity analysis } \\
\hline High-quality study & $52 \%$ & $-3.87(-4.47,-3.28)$ & $0 \%$ & $-2.71(-3.00,-2.42)$ & $76 \%$ & $-1.25(-1.73,-0.77)$ & $75 \%$ & $-0.36(-0.64,-0.09)$ \\
\hline Age $\leq 50$ y & $36 \%$ & $-3.77(-4.27,-3.27)$ & $0 \%$ & $-2.69(-3.06,-2.32)$ & $0 \%$ & $-1.05(-1.24,-0.86)$ & $79 \%$ & $-0.35(-0.72,0.01)^{*}$ \\
\hline$>50 y$ & $94 \%$ & $-5.35(-11.01,0.31)^{*}$ & $0 \%$ & $-1.85(-2.18,-1.51)$ & $86 \%$ & $-1.67(-3.62,0.28)^{*}$ & $0 \%$ & $-0.55(-0.75,-0.34)$ \\
\hline Stent $<6 \mathrm{~F}$ & $54 \%$ & $-4.92(-7.29,-2.54)$ & $0 \%$ & $-2.74(-3.03,-2.44)$ & $83 \%$ & $-1.70(-3.75,0.34)^{*}$ & $0 \%$ & $-0.59(-0.82,-0.37)$ \\
\hline$\geq 6 \mathrm{~F}$ & $95 \%$ & $-4.15(-5.79,-2.50)$ & $99 \%$ & $-0.08(-0.31,0.15)^{*}$ & $96 \%$ & $-0.29(-0.45,-0.14)$ & $75 \%$ & $-0.33(-0.65,-0.02)$ \\
\hline Tamsulosin & $95 \%$ & $-4.32(-6.83,-1.82)$ & $99 \%$ & $-0.52(-0.78,-0.27)$ & $95 \%$ & $-0.14(-0.29,0.01)^{*}$ & $81 \%$ & $-0.4 \mathrm{I}(-0.9 \mathrm{I}, 0.09)^{*}$ \\
\hline Other $\alpha$-blockers ${ }^{\#}$ & $58 \%$ & $-3.94(-4.98,-2.90)$ & $20 \%$ & $-2.66(-3.35,-1.97)$ & $89 \%$ & $-1.48(-2.62,-0.34)$ & $18 \%$ & $-0.47(-0.66,-0.29)$ \\
\hline \multicolumn{9}{|l|}{ Indirect comparison } \\
\hline Tamsulosin vs other $\alpha$-blockers & & $-0.38(-3.09,2.33)^{*}$ & & $2.14(\mathrm{I} .4 \mathrm{I}, 2.88)$ & & $1.34(0.19,2.49)$ & & $0.06(-0.39,0.5 \mathrm{I})^{*}$ \\
\hline
\end{tabular}

Notes: *No significant difference was found. ${ }^{*}$ Other $\alpha$-blockers included alfuzosin and terazosin.

Abbreviations: $y$, years; $\mathrm{MD}$, mean difference; $\mathrm{Cl}$, confidence interval. 


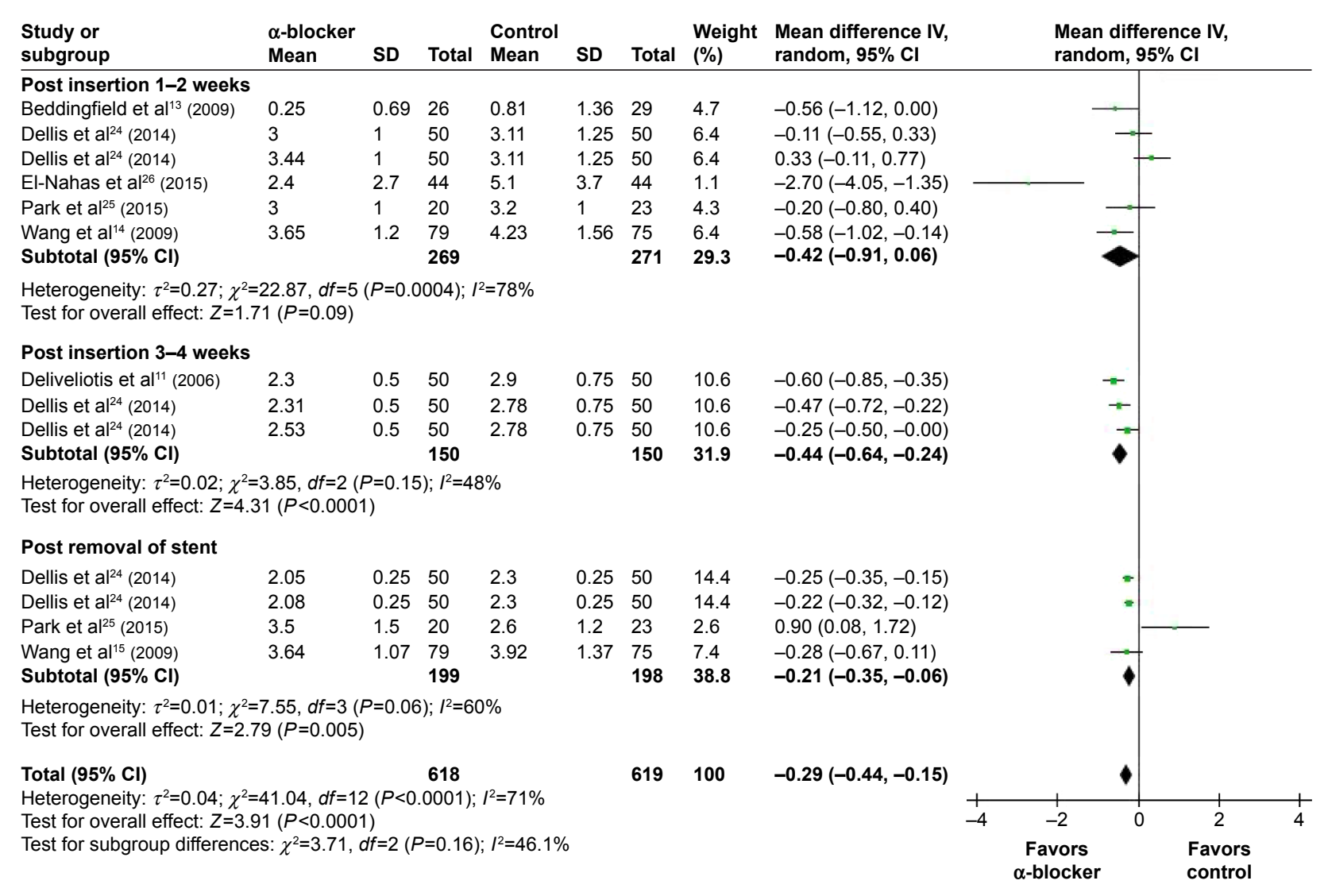

Figure 6 Meta-analysis results of sexual matters score.

Abbreviations: $95 \% \mathrm{Cl}, 95 \%$ confidence interval; $d f$, degrees of freedom.

\section{Additional problems score}

Only two trials reported the mean additional problems score during post-insertion 1-2 weeks, ${ }^{13,14}$ and meta-analysis result showed no significant difference between $\alpha$-blockers and control $\left(I^{2}=91 \%, \mathrm{MD}=-1.50,95 \% \mathrm{CI},-4.42,1.43\right.$, $P=0.32$ ).

\section{Quality of life score}

Compared with control, $\alpha$-blockers significantly decreased the mean quality of life score ( $I^{2}=96 \%, \mathrm{MD}=-1.38,95 \%$ $\mathrm{CI},-1.96,-0.79, P<0.00001)$. Subgroup analysis according to outcome measured time showed that $\alpha$-blockers significantly improved the quality of life score during postinsertion $1-2$ weeks by a mean of -1.50 (95\% CI, -2.39 to -0.62$),{ }^{14,16,21,22,24,25} 3-4$ weeks by a mean of $-1.77(95 \%$ CI, -3.11 to -0.42$),{ }^{16,17,19,23,24}$ and post-removal by a mean of -0.44 ( $95 \% \mathrm{CI},-0.70$ to -0.19$).{ }^{24,25}$

\section{Others}

Three trials reported data of patients with pain, ${ }^{11,14,24}$ and their results showed that $\alpha$-blockers significantly decreased the incidence of pain compared with control $(36.68 \%$ vs $\left.46.67 \%, P^{2}=0 \%, \mathrm{RR}=0.78,95 \% \mathrm{CI},-0.63,0.97, P=0.03\right)$.

Meta-analysis also revealed a superiority of $\alpha$-blockers in aspects of irritative symptoms score $\left(I^{2}=99 \%, \mathrm{MD}=-5.43\right.$, 95\% CI, $-10.09,-0.77, P=0.02)$ and obstructive symptoms score $\left(I^{2}=99 \%, \mathrm{MD}=-4.27,95 \% \mathrm{CI},-6.93,-1.61\right.$, $P=0.002)$.

\section{Discussion}

As $\alpha$-blockers were reported to be effective in the management after US insertion, many studies were conducted, and this study is an updated meta-analysis including the latest published articles. The study confirmed the efficacy of $\alpha$-blockers in improving USSs, and, to our knowledge, it is also the first to qualitatively investigate the important clinical factors including stent duration, patient age, stent size, and $\alpha$-blocker type, and to suggest the potential superiority of alfuzosin and terazosin compared to tamsulosin.

To judge the therapeutic effect of $\alpha$-blockers, International Prostate Symptom Score (IPSS) ${ }^{28}$ The Short-Form 36 Health Status Questionnaire (SF 36), ${ }^{29}$ the EuroQol, ${ }^{30}$ and the USSQ were used independently or complementarily in the included trials. Among them, USSQ was a self-administrated 
multidimensional measure presented by Joshi et $\mathrm{al}^{31}$ after they brought to attention the need for a validated questionnaire of USSs. It has proven to be a sensitive and stent-specific measuring tool. ${ }^{32}$ Therefore, the meta-analysis mainly adopted items from USSQ as outcome measures to compare the efficacy of $\alpha$-blocker in improving USSs.

Compared to control, additional administrated $\alpha$-blockers significantly improved urinary symptom score and pain index score, and they had substantial positive effects on the general health index score, sexual matters score, pain incidence, irritative symptoms score, and obstructive symptoms score, but had no effect on work performance score and additional problems score. Previous studies had found an association between USSs and patients' quality of life, ${ }^{32,33}$ and this study also indicated the association, as the improved quality of life score may be mainly contributed by the improved urinary symptom and pain index. Meanwhile, after including 16 RCTs of 1,489 cases, such a meta-analysis in randomeffects models could, in a conservative manner, not only confirm the overall effects but also enable the investigation of clinical heterogeneity and different factors across the trials through the statistical methods of subgroup analysis and sensitive analysis. ${ }^{34}$

To investigate heterogeneity in the major outcome measures, first we omitted relatively low-quality trials, and the values of $I^{2}$ were to some extent reduced. Meanwhile, the outcome of urinary symptom score, pain index score, general health index, and sexual matters score did not obviously change, indicating a reliable and stable efficacy of $\alpha$-blockers in improving USSQ. In order to ensure sufficiently statistical test power, an $80 \%$ power to detect a $15 \%$ difference in the urinary symptom score, a $30 \%$ difference in the pain index score, and a $25 \%$ difference in the general health index, ${ }^{24}$ further subgroup and sensitivity analyses of outcome measured time, patient age, stent diameter, and $\alpha$-blocker types were conducted based on a sample size of $>50$ patients in each group.

It has been reported that different durations of an indwelling stent would induce different influences on patients ${ }^{35}$ and a longer duration can improve the overall tolerance. ${ }^{5,6}$ Our study included trials with duration of stent insertion as well as drug administration from 8 days to 6 months, and researchers usually applied USSQ to patients one or more times during the period from insertion and even after removal. Therefore, considering the stent duration, drug administration, and measured time, a subgroup analysis during post-insertion 1-2 weeks, 3-4 weeks, and post-removal was primarily conducted. The results showed that $\alpha$-blockers had a wide role in improving both short-term and long-term USSs, and also had an effect even after stent removal, while they had little influence on short-term pain index, post-removal general health, and sexual matters.

As presented in the study of Wang et al, ${ }^{14}$ patients with age $>50$ years had very different baseline IPSSs but similar USSQ compared with those aged $\leq 50$ years. Regretfully, although the study distinguished sex (male/female) and age $(<50 / \geq 50$ years), the authors did not make any comments on the issues. Confusingly, a rough analysis of three trials in our study showed that for patients $\geq 50$ years $\alpha$-blockers improved not urinary symptom score but sexual matters score. ${ }^{11,14,25}$ Perhaps, the possibility of worse baseline condition of urinary symptoms in patients $\geq 50$ years combined with additional burden of the stent was sometimes beyond the ability of the $\alpha$-blocker. ${ }^{36}$

Some previous studies compared different stent diameters of $4.8 \mathrm{~F}$ and $6 \mathrm{~F}$, and demonstrated that there was no significant difference in the aspects of pain, urinary symptoms, and quality of life, but the $4.8 \mathrm{~F}$ stent was associated with a higher frequency of distal migration and dislodging. ${ }^{37-39}$ However, the studies failed to reach the estimated sample size of 50 cases in each group, and our study revealed that patients with a stent $\geq 6 \mathrm{~F}$ would suffer much more pain than with a stent $<6 \mathrm{~F}$ as $\alpha$-blocker was found to fail to reduce the pain score index in the former cases.

Also, our meta-analysis is the first to investigate the difference between tamsulosin (a highly selective $\alpha-1 \mathrm{~A}$ and $\alpha-1 \mathrm{D}$ adrenoceptor blocker) and other $\alpha$-blockers including alfuzosin and terazosin (selective $\alpha-1$ adrenoceptor blockers). Our results indicated that the latter achieved better improvement in the pain index and general health index score. In the absence of any elucidated mechanism of each symptom, ${ }^{40}$ the difference between a highly selective $\alpha-1$ blocker and a selective $\alpha-1$ blocker may be due to the 1) the different location of $\alpha$ receptor in ureter and bladder trigone, ${ }^{3,5} 2$ ) the different pharmacological and physiological actions of $\alpha$ blockers and receptors, and 3) the different dosage and course of the drugs.

The limitations of our study are as follows. 1) In our study design, four of the included RCTs were of relatively poor quality and seven of them adopted a control other than placebo. 2) As mentioned above, many clinical factors and any underlying ureteral disease would have influenced the outcomes, and also different patient characteristics had a negative influence on the overall estimates. 3) Although different methods were used to identify and minimize the clinical factors, all these may still induce unavoidable heterogeneity 
and bias. 4) As a study of positive results seems to be easier to get published than a negative one, publication bias might always exist. 5) For safety, as complications related to such $\alpha$-blockers were stated to be mild and rare, ${ }^{5}$ our study did not present much data on this. 6) Though the material and the site of stent distal end have been demonstrated to be very important factors, ${ }^{41,42}$ our study did not involve the terms due to lack of information. 7) Finally, several recent studies have suggested that a combination therapy by $\alpha$-blockers and antimuscarinic agents may be better than monotherapy with $\alpha$-blockers alone, but due to lack of sufficient RCTs, our study did not involve this issue.

\section{Conclusion}

An $\alpha$-blocker was found to be effective in treating USSs, as it improved the major indexes of USSQ post-insertion or post-removal. Alfuzosin and terazosin seemed to be better than tamsulosin, but this could not be verified due to lack of direct comparison studies currently.

\section{Disclosure}

The authors report no conflicts of interest in this work.

\section{References}

1. Zimskind PD, Fetter TR, Wilkerson JL. Clinical use of long-term indwelling silicone rubber ureteral splints inserted cystoscopically. J Urol. 1967;97(5):840-844.

2. Nabi G, Cook J, N'Dow J, McClinton S. Outcomes of stenting after uncomplicated ureteroscopy: systematic review and meta-analysis. BMJ. 2007;334(7593):572.

3. Lamb AD, Vowler SL, Johnston R, Dunn N, Wiseman OJ. Meta-analysis showing the beneficial effect of $\alpha$-blockers on ureteric stent discomfort. BJU Int. 2011;108(11):1894-1902.

4. Haleblian G, Kijvikai K, de la Rosette J, Preminger G. Ureteral stenting and urinary stone management: a systematic review. J Urol. 2008; 179(2):424-430.

5. Zhou L, Cai X, Li H, Wang KJ. Effects of $\alpha$-blockers, antimuscarinics, or combination therapy in relieving ureteral stent-related symptoms: a meta-analysis. J Endourol. 2015;29(6):650-656.

6. Irani J, Siquier J, Pirès C, Lefebvre O, Doré B, Aubert J. Symptom characteristics and the development of tolerance with time in patients with indwelling double-pigtail ureteric stents. BJU Int. 1999;84(3): 276-279.

7. Joshi HB, Stainthorpe A, MacDonagh RP, Keeley FX Jr, Timoney AG, Barry MJ. Indwelling ureteral stents: evaluation of symptoms, quality of life and utility. J Urol. 2003;169(3):1065-1069.

8. Zhu L, Feng Z, Zhou Q. Personalized therapeutics of $\alpha$ 1-blockers in patients with lower urinary tract symptoms suggestive of benign prostatic hyperplasia. Clin Interv Aging. 2015;10:621-622.

9. Russo A, La Croce G, Capogrosso P, et al. Latest pharmacotherapy options for benign prostatic hyperplasia. Expert Opin Pharmacother. 2014;15(16):2319-2328.

10. Thorpe A, Neal D. Benign prostatic hyperplasia. Lancet. 2003;361(9366): 1359-1367.

11. Deliveliotis C, Chrisofos M, Gougousis E, Papatsoris A, Dellis A, Varkarakis IM. Is there a role for alpha 1-blockers in treating double-J stent-related symptoms? Urology. 2006;67(1):35-39.
12. Damiano R, Autorino R, De Sio M, Giacobbe A, Palumbo IM, D'Armiento M. Effect of tamsulosin in preventing ureteral stent-related morbidity: a prospective study. J Endourol. 2008;22(4):651-656.

13. Beddingfield R, Pedro RN, Hinck B, Kreidberg C, Feia K, Monga M. Alfuzosin to relieve ureteral stent discomfort: a prospective, randomized, placebo controlled study. J Urol. 2009;181(1):170-176.

14. Wang CJ, Huang SW, Chang CH. Effects of specific $\alpha-1 \mathrm{~A} / 1 \mathrm{D}$ blocker on lower urinary tract symptoms due to double-J stent: a prospectively randomized study. Urol Res. 2009;37(3):147-152.

15. Wang CJ, Huang SW, Chang CH. Effects of tamsulosin on lower urinary tract symptoms due to double-J stent: a prospective study. Urol Int. 2009;83(1):66-69.

16. Navanimitkul N, Lojanapiwat B. Efficacy of tamsulosin $0.4 \mathrm{mg} / \mathrm{day}$ in relieving double-J stent-related symptoms: a randomized controlled study. J Int Med Res. 2010;38(4):1436-1441.

17. Shelbaia A, Elnashar A. Role of tamsulosin in improving double-J ureteric stent-related symptoms. Afr J Urol. 2011;17(4):111-114.

18. Mokhtari G, Shakiba M, Ghodsi S, Farzan A, Heidari Nejad S, Esmaeili S. Effect of terazosin on lower urinary tract symptoms and pain due to double-J stent: a double-blind placebo-controlled randomized clinical trial. Urol Int. 2011;87(1):19-22.

19. Kuyumcuoglu U, Eryildirim B, Tuncer M, Faydaci G, Tarhan F, Ozgül A. Effectiveness of medical treatment in overcoming the ureteral double-J stent related symptoms. Can Urol Assoc J. 2012;6(6): e234-e237.

20. Nazim SM, Ather MH. Alpha-blockers impact stent-related symptoms: a randomized, double-blind, placebo-controlled trial. J Endourol. 2012;26(9):1237-1241.

21. Shalaby E, Ahmed A, Maarouf A, Yahia I, Ali M, Ghobish A. Randomized controlled trial to compare the safety and efficacy of tamsulosin, solifenacin, and combination of both in treatment of double-j stentrelated lower urinary symptoms. Adv Urol. 2013;2013:752382.

22. Tehranchi A, Rezaei Y, Khalkhali H, Rezaei M. Effects of terazosin and tolterodine on ureteral stent related symptoms: a double-blind placebocontrolled randomized clinical trial. Int Braz J Urol. 2013;39(6): 832-840.

23. Singh I, Tripathy S, Agrawal V. Efficacy of tamsulosin hydrochloride in relieving "double-J ureteral stent-related morbidity": a randomized placebo controlled clinical study. Int Urol Nephrol. 2014;46(12): 2279-2283.

24. Dellis AE, Keeley FX Jr, Manolas V, Skolarikos AA. Role of $\alpha$-blockers in the treatment of stent-related symptoms: a prospective randomized control study. Urology. 2014;83(1):56-62.

25. Park J, Yoo C, Han DH, Shin DW. A critical assessment of the effects of tamsulosin and solifenacin as monotherapies and as a combination therapy for the treatment of ureteral stent-related symptoms: a $2 \times 2$ factorial randomized trial. World J Urol. 2015;33(11):1833-1840.

26. El-Nahas AR, Tharwat M, Elsaadany M, Mosbah A, Gaballah MA. A randomized controlled trial comparing alpha blocker (tamsulosin) and anticholinergic (solifenacin) in treatment of ureteral stent-related symptoms. World J Urol. Epub 2015 Oct 9:1-6.

27. Higgins JPT, Altman DG, Gøtzsche PC, et al; Cochrane Bias Methods Group; Cochrane Statistical Methods Group. The Cochrane Collaboration's tool for assessing risk of bias in randomised trials. BMJ. 2011;343:d5928.

28. Ushijima S, Ukimura O, Okihara K, Mizutani Y, Kawauchi A, Miki T. Visual analog scale questionnaire to assess quality of life specific to each symptom of the International Prostate Symptom Score. J Urol. 2006; 176(2):665-671.

29. Ziebland $\mathrm{S}$. The short form 36 health status questionnaire: clues from the Oxford region's normative data about its usefulness in measuring health gain in population surveys. J Epidemiol Community Health. 1995;49(1):102-105.

30. Group TEQ. EuroQol-a new facility for the measurement of healthrelated quality of life. Health Policy. 1990;16(3):199-208.

31. Joshi HB, Okeke A, Newns N, Keeley FX Jr, Timoney AG. Characterization of urinary symptoms in patients with ureteral stents. Urology. 2002;59(4):511-516. 
32. Joshi HB, Newns N, Stainthorpe A, MacDonagh RP, Keeley FX Jr, Timoney AG. Ureteral stent symptom questionnaire: development and validation of a multidimensional quality of life measure. J Urol. 2003; 169(3):1060-1064.

33. Coyne KS, Wein AJ, Tubaro A, et al. The burden of lower urinary tract symptoms: evaluating the effect of LUTS on health-related quality of life, anxiety and depression: EpiLUTS. BJU Int. 2009;103(s3):4-11.

34. Hahn S, Williamson PR, Hutton JL, Garner P, Flynn EV. Assessing the potential for bias in meta-analysis due to selective reporting of subgroup analyses within studies. Stat Med. 2000;19(24):3325-3336.

35. Damiano R, Oliva A, Esposito C, De Sio M, Autorino R, D'Armiento M. Early and late complications of double pigtail ureteral stent. Urol Int. 2002;69(2):136-140.

36. Malone-Lee J, Shaffu B, Anand C, Powell C. Tolterodine: superior tolerability than and comparable efficacy to oxybutynin in individuals 50 years old or older with overactive bladder: a randomized controlled trial. J Urol. 2001;165(5):1452-1456.

37. Erturk E, Sessions A, Joseph JV. Impact of ureteral stent diameter on symptoms and tolerability. J Endourol. 2003;17(2):59-62.
38. Damiano R, Autorino R, De Sio M, et al. Does the size of ureteral stent impact urinary symptoms and quality of life? A prospective randomized study. Eur Urol. 2005;48(4):673-678.

39. Candela JV, Bellman GC. Ureteral stents: impact of diameter and composition on patient symptoms. J Endourol. 1997;11(1):45-47.

40. Miyaoka R, Monga M. Ureteral stent discomfort: etiology and management. Indian J Urol. 2009;25(4):455.

41. Park HK, Paick SH, Kim HG, Lho YS, Bae S. The impact of ureteral stent type on patient symptoms as determined by the ureteral stent symptom questionnaire: a prospective, randomized, controlled study. J Endourol. 2015;29(3):367-371.

42. Lee SJ, Yoo C, Oh CY, et al. Stent position is more important than $\alpha$-blockers or anticholinergics for stent-related lower urinary tract symptoms after ureteroscopic ureterolithotomy: a prospective randomized study. Korean J Urol. 2010;51(9):636-641.

\section{Publish your work in this journal}

Drug Design, Development and Therapy is an international, peerreviewed open-access journal that spans the spectrum of drug design and development through to clinical applications. Clinical outcomes, patient safety, and programs for the development and effective, safe, and sustained use of medicines are a feature of the journal, which has also been accepted for indexing on PubMed Central. The manuscript management system is completely online and includes a very quick and fair peer-review system, which is all easy to use. Visit http://www.dovepress.com/testimonials.php to read real quotes from published authors.

Submit your manuscript here: http://www.dovepress.com/drug-design-development-and-therapy-journal 